\title{
Zn-Doping Dependence of Stripe Order in La1.905Ba0.095CuO4
}

Journal of Superconductivity and Novel Magnetism

Incorporating Novel Magnetism

ISSN 1557-1939

Volume 24

Number 3

J Supercond Nov Magn (2011) 24:1229-1233

DOI $10.1007 /$

s10948-010-1122-0

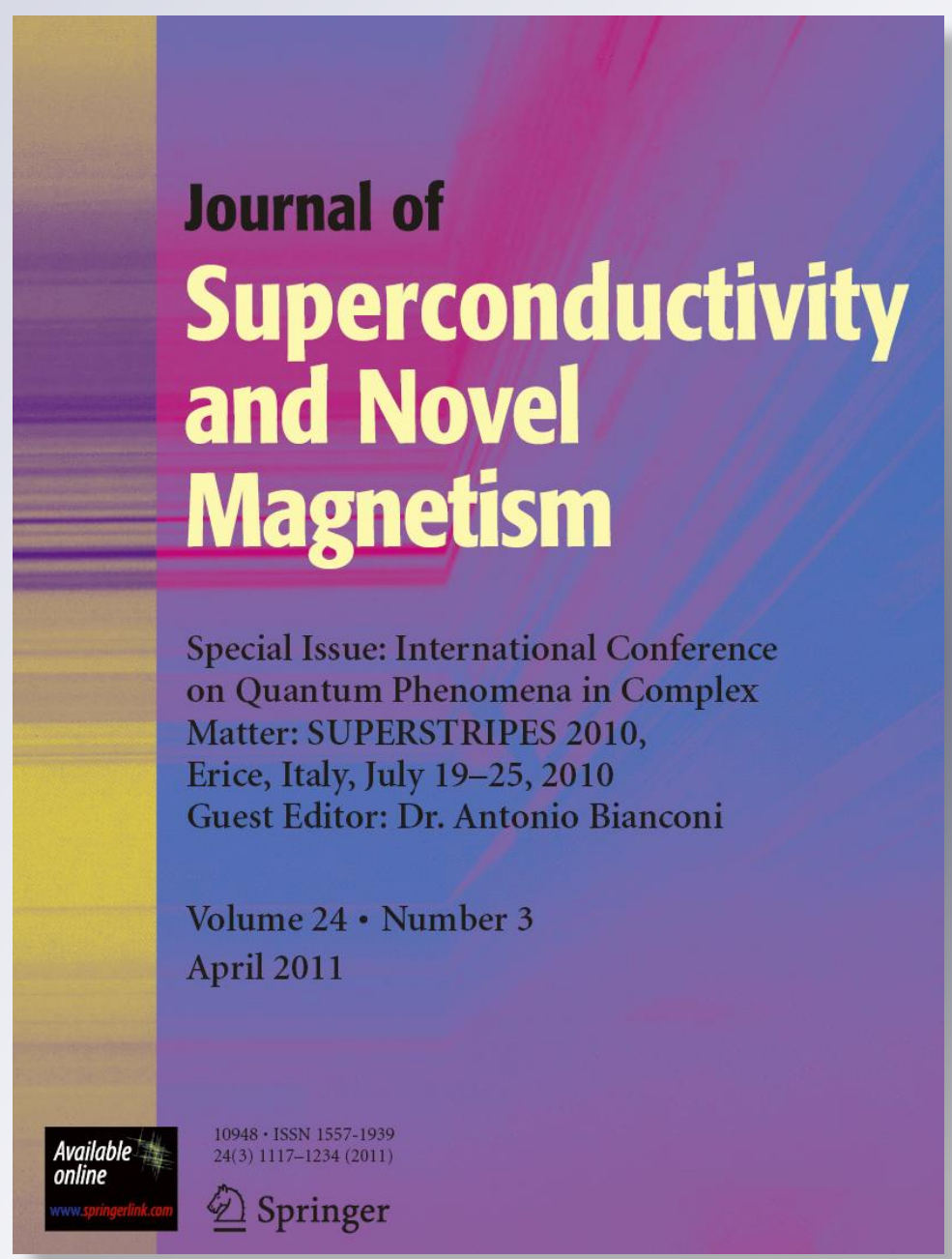

Springer 
Your article is protected by copyright and all rights are held exclusively by Springer Science+Business Media, LLC. This e-offprint is for personal use only and shall not be selfarchived in electronic repositories. If you wish to self-archive your work, please use the accepted author's version for posting to your own website or your institution's repository. You may further deposit the accepted author's version on a funder's repository at a funder's request, provided it is not made publicly available until 12 months after publication. 


\title{
Zn-Doping Dependence of Stripe Order in $\mathrm{La}_{1.905} \mathrm{Ba}_{0.095} \mathrm{CuO}_{4}$
}

\author{
M. Hücker • M.v. Zimmermann • Z.J. Xu • J.S. Wen • \\ G.D. Gu $\cdot$ W. Tian $\cdot$ J. Zarestky $\cdot$ J.M. Tranquada
}

Received: 9 December 2010 / Accepted: 26 December 2010 / Published online: 28 January 2011

(C) Springer Science+Business Media, LLC (outside the USA) 2011

\begin{abstract}
The effect of $\mathrm{Zn}$-doping on the stripe order in $\mathrm{La}_{1.905} \mathrm{Ba}_{0.095} \mathrm{CuO}_{4}$ has been studied by means of $\mathrm{x}$-ray and neutron diffraction as well as magnetization measurements. While $1 \% \mathrm{Zn}$ leads to an increase of the spin stripe order, it unexpectedly causes a wipe out of the visibility of the charge stripe order. A magnetic field of 10 Tesla applied along the c-axis has no reversing effect on the charge order. We compare this observation with the Zn-doping dependence of the crystal structure, superconductivity, and normal state magnetism.
\end{abstract}

Keywords Stripes $\cdot$ Cuprates $\cdot$ Zn doping

\section{Introduction}

Numerous studies on high- $T_{c}$ superconductors have demonstrated that the electronic properties of the $\mathrm{CuO}_{2}$ layers are extremely sensitive to impurity doping, such as the substitution of $\mathrm{Cu}$ with $3 d$-transition metals. The most obvious effect is the suppression of the superconductivity (SC) [1]. In

The work at Brookhaven was supported by the Office of Science, U.S. Department of Energy under Contract No. DE-AC02-98CH10886. J.S.W. and Z.J.X. are supported by the Center for Emergent Superconductivity, an Energy Frontier Research Center funded by the US DOE, Office of Basic Energy Sciences.

M. Hücker $(\bowtie)$ · Z.J. Xu · J.S. Wen · G.D. Gu · J.M. Tranquada Brookhaven National Laboratory, Upton, NY 11973, USA e-mail: huecker@bnl.gov

M.v. Zimmermann

Hamburger Synchrotronstrahlungslabor HASYLAB at Deutsches

Elektronen-Synchrotron DESY, Hamburg 22603, Germany

W. Tian · J. Zarestky

Ames Laboratory, Ames, IA 50011, USA addition, in several cases a ground state with static or slowly fluctuating antiferromagnetic (AF) correlations is stabilized or enhanced [2-4]. In $\mathrm{Zn}$-doped $\mathrm{La}_{2-x} \mathrm{Sr}_{x} \mathrm{CuO}_{4}$ (LSCO) the competing AF order is incommensurate, and similar to the magnetism in charge $(\mathrm{CO})$ and spin $(\mathrm{SO})$ stripe ordered $\mathrm{La}_{2-x} \mathrm{Ba}_{x} \mathrm{CuO}_{4}$ (LBCO) [5-8]. For many years it was unclear whether the two ground states are indeed identical, due to lack of evidence of $\mathrm{CO}$ in $\mathrm{Zn}$-doped LSCO. Although the presence of $\mathrm{CO}$ has been expected [9], only recently it was confirmed when weak CO-reflections were identified in $\mathrm{Zn}$ doped LSCO with $x \sim 1 / 8$ [10].

However, numerous experiments suggest that for symmetry reasons stripes are pinned more effectively in the lowtemperature tetragonal (LTT) phase (space group $\mathrm{P} 4_{2} / \mathrm{ncm}$ ) of LBCO and related compounds such as $\mathrm{La}_{2-x-y} \mathrm{Nd}_{y} \mathrm{Sr}_{x}$ $\mathrm{CuO}_{4}$ [7] than in the low-temperature orthorhombic (LTO) phase of LSCO (Bmab). The LTT phase lifts the degeneracy of the two orthogonal directions parallel to the $\mathrm{CuO}_{2}$ square lattice which promotes the pinning of stripes parallel to the $\mathrm{Cu}-\mathrm{O}-\mathrm{Cu}$ bonds, while the LTO phase lifts the degeneracy of the diagonal lattice directions which leaves all $\mathrm{Cu}-\mathrm{O}-\mathrm{Cu}$ bonds equivalent. However, high pressure experiments revealed that exactly at $x=1 / 8$, which is the hole content most favorable for stripe order [11], CO can spontaneously break the in-plane fourfold rotational symmetry even in the high-temperature tetragonal (HTT) phase $\left(I_{4} / \mathrm{mmm}\right)$ with average flat $\mathrm{CuO}_{2}$ planes [12], possibly with help of local lattice distortions. Whether these distortions are LTT or LTO-like is unknown. In [13] it is reported that high $(\mathrm{Zn}, \mathrm{Mg})$-concentrations in $\mathrm{La}_{2} \mathrm{CuO}_{4} \mathrm{ul}$ timately stabilize the LTT phase. Thus, beside the effect of depressed SC around Zn, small amounts of Zn could locally promote LTT-like distortions that further support the pinning of stripes in the LTO phase [14]. 
An interesting discussion of stripe pinning mechanisms in ( $\mathrm{Zn}, \mathrm{Ga}, \mathrm{Fe}$ )-doped LSCO at $x \sim 1 / 8$ is given in [10]. Although any of these defects could stabilize CO, especially large effects on both $\mathrm{CO}$ and $\mathrm{SO}$ are observed for Fedoping which introduces large magnetic spin defects $(S=$ $5 / 2$ ) [10]. An interesting feature of [10] is that $\mathrm{Zn}$ stabilizes a weak $\mathrm{CO}$, but does not enhance the weak SO already present in pure LSCO. Earlier observations near optimum doping indicated a $\mathrm{Zn}$-induced stabilization, in particular for the SO [5, 6].

To further elucidate the possible mechanisms by which stripes can be stabilized, we decided to study the effect of Zn-doping on LBCO, i.e., a system where $\mathrm{CO}$ and $\mathrm{SO}$ forms naturally without impurity doping [8]. Two crystals with $x=0.095$ were studied, one with $0 \% \mathrm{Zn}$ (LBCO95) and the other with $1 \% \mathrm{Zn}$ (LBCZO95). Note that the crystals low$T$ structure is not perfectly LTT, but assumes the so-called less-orthorhombic low-temperature (LTLO) phase (Pccn), which is an intermediate phase between LTO and LTT with reduced orthorhombic strain. However, at base temperature the remaining strain is extremely small, and the crystals are very close to LTT [11]. In LBCO95 stripe order is much weaker than at $x=1 / 8$ [11], which enables us to identify both possible scenarios: The enhancement or the degradation of $\mathrm{CO}$ and $\mathrm{SO}$ order by $\mathrm{Zn}$-doping. Indeed we found a $\mathrm{Zn}$-induced increase of the SO, which led us to expect that the $\mathrm{CO}$ order will increase as well. To our surprise, however, the opposite is true in our LBCZO95 crystal. Possible reasons will be discussed.

\section{Experimental}

The LBCO95 and LBCZO95 crystals were grown with the traveling-solvent floating-zone technique. The stripe order, structure and resistivity of the LBCO95 crystal were subject of two further studies $[11,15]$. The $\mathrm{x}$-ray diffraction (XRD) experiments were performed with a triple-axis diffractometer at wiggler beamline BW5 at DESY, using $100 \mathrm{keV}$ photons in transmission geometry; the neutron diffraction (ND) experiments with triple-axis spectrometer HB1A at the High Flux Isotope Reactor, Oak Ridge National Laboratory, using beam collimations of $48^{\prime}-48^{\prime}-\mathrm{S}-40^{\prime}-136^{\prime}$ with a fixed incident energy $E_{i}=14.6 \mathrm{meV}$. Scattering vectors $\mathbf{Q}=(h, k, \ell)$ are specified in units of $(2 \pi / a, 2 \pi / a, 2 \pi / c)$, where $a \simeq$ $3.78 \AA$ and $c \simeq 13.2 \AA$ are the lattice parameters of the HTT phase. The DC magnetic susceptibility $\chi=M / H$ in the SC state as well as in the normal state has been analyzed with a SQUID-magnetometer for $H$ parallel and perpendicular to the $\mathrm{CuO}_{2}$ planes.

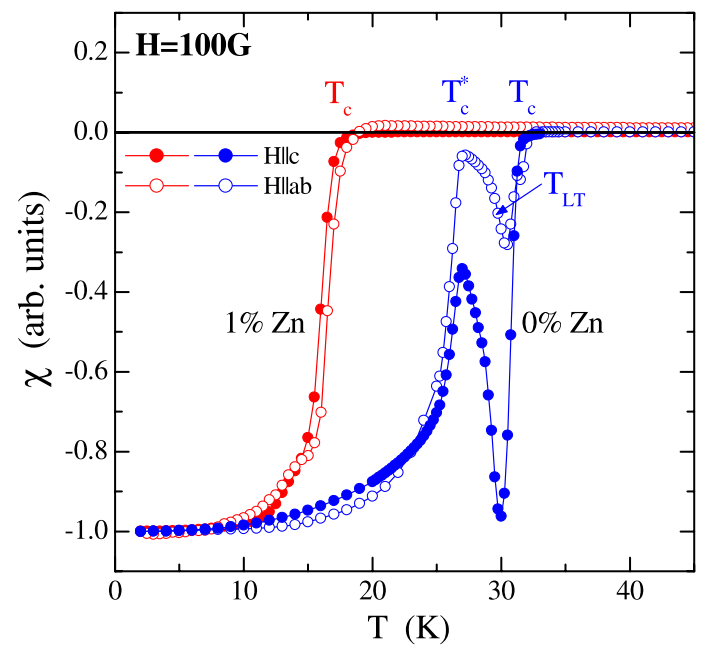

Fig. 1 (Color online) Normalized field cooled susceptibility vs. $T$ of pure and $\mathrm{Zn}$-doped $\mathrm{La}_{1.905} \mathrm{Ba}_{0.095} \mathrm{CuO}_{4}$ at $H=100 \mathrm{G}$ applied parallel $a b$-plane and $c$-axis

\section{Results}

\subsection{Superconductivity}

First we look at the SC properties which have been characterized with $\chi(T)$ measurements at $H=100 \mathrm{G}$, and are displayed in Fig. 1 on normalized $\chi$ scale. Obviously, LBCO95 shows a peculiar sequence of transitions, discussed in [11]. At $T_{c} \simeq 32 \mathrm{~K}$ the sample turns bulk SC in the LTO phase. Just slightly below that temperature, at $T \simeq 30 \mathrm{~K}$, the diamagnetic signal collapses when the transitions to $\mathrm{CO}$ and to the LTLO phase occur; see next sections. At $T_{c}^{*} \simeq 27 \mathrm{~K}$ occurs a second SC transition that coincides with the completion of the LTO $\rightarrow$ LTLO transition [11]. The LBCZO95 crystal shows a much simpler behavior. There is only one SC transition at $T_{c} \simeq 18 \mathrm{~K}$, which is pushed below those in $\mathrm{LBCO} 95$ by the $\mathrm{Zn}$. Moreover, there is no clear signature of the LTO $\leftrightarrow$ LTLO transition, except for a small hump at $\sim 15 \mathrm{~K}$. As will be discussed in Sect. 3.2 this hump may coincide with the completion of the LTO $\rightarrow$ LTLO transition in $\mathrm{LBCZO} 95$.

\subsection{Crystal Structure}

Next we focus on the crystal structure. For the present work it is sufficient to analyze the LTO $\leftrightarrow$ LTLO transition, by following the $T$ dependence of the $(3,0,0)$ peak intensity; see the reciprocal lattice in Fig. 2(b). The XRD data in Fig. 2(a) show a significant change with $\mathrm{Zn}$. While the transition is sharp in LBCO95, it is very broad in LBCZO95, even though the onset temperatures are about the same. How does this affect the CO? (The results for LBCO95 were measured on the identical sample as in [11], but with different cryostat and scattering geometry, explaining the slightly different $T_{\mathrm{LT}}$ and width of the transition.) 


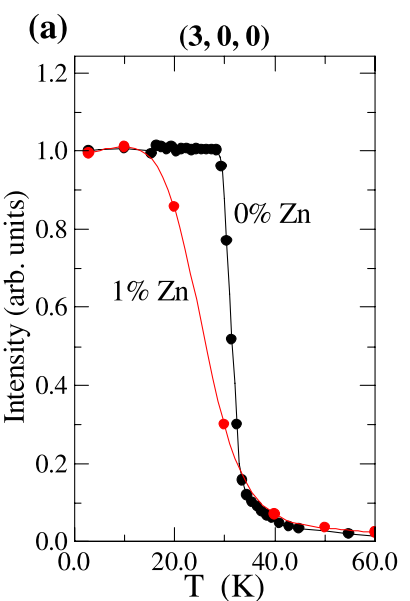

(c)

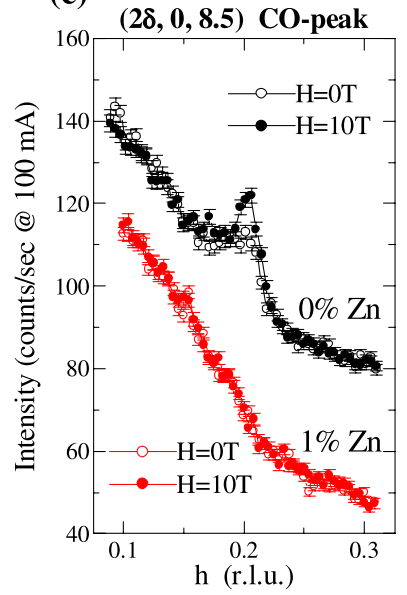

(b)

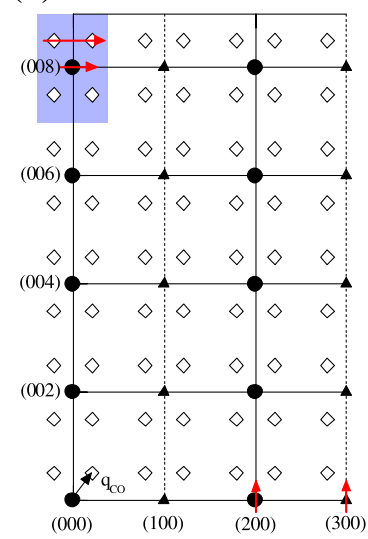

(d)

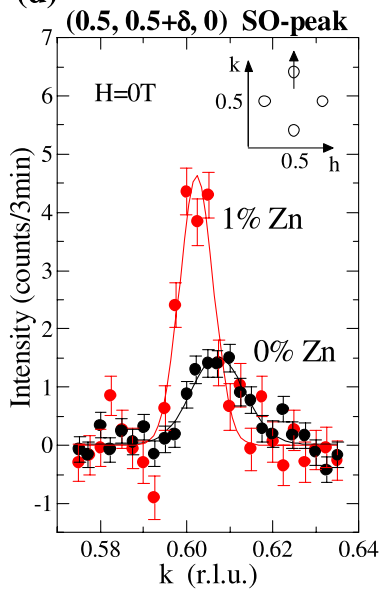

Fig. 2 (Color online) Crystal structure and stripe order of pure and Zn-doped $\mathrm{La}_{1.905} \mathrm{Ba}_{0.095} \mathrm{CuO}_{4}$. (a) Normalized integrated intensity from $k$-scans through $(3,0,0)$ LTLO-peak. (b) Reciprocal lattice of the $(h, 0, \ell)$-plane in the LTLO phase. $(\bullet) \mathrm{Bragg},(\diamond) \mathrm{CO}$, and $(\Delta)$ LTLO-reflections. Red arrows indicate typical scans, and blue rectangle a studied mesh. $\mathrm{q}_{\mathrm{CO}}$ indicates $\mathrm{CO}$ ordering wave vector $(2 \delta, 0,0.5)$. (c) $h$-scans through $(2 \delta, 0,8.5) \mathrm{CO}$-peak at $T=3 \mathrm{~K}$ and $H=0 \mathrm{~T}$ and $10 \mathrm{~T}$ applied parallel $c$-axis. Data for $0 \% \mathrm{Zn}$ are shifted by 30 counts/s. (d) $k$-scans through $(0.5,0.5+\delta, 0) \mathrm{SO}$-peak. Data show the difference between $10 \mathrm{~K}$ and $50 \mathrm{~K}$. Inset: SO-peaks in $(h, k, 0)$-plane

\subsection{Spin and Charge Stripe Order}

The $\mathrm{CO}$ leads to weak reflections with ordering wave vector $q_{\mathrm{CO}}$, as indicated in Fig. 2(b). In Fig. 2(c) we present $h$-scans at $3 \mathrm{~K}$ through $q=(2 \delta, 0,8.5)$, which is among the most intense $\mathrm{CO}$ reflections. While for LBCO 95 a CO-peak is clearly observed, in LBCZO95 it is not identifiable. This is in stark contrast to the SO-peak in Fig. 2(d), which increases with $\mathrm{Zn}$-doping. When applying a field of 10 Tesla parallel to the $c$-axis, the CO-peak in LBCO95 grows while it has no effect on LBCZO95. To check whether Zn-doping caused the CO-peak position to shift, we performed scans in a wider range, and indeed found some broad peaks that are displayed in Fig. 3(a). However, these weakly $T$ depen-

dent features turned out to be the tails of the butterfly like diffuse scattering around the nearby $(0,0,8)$ Bragg reflection; see Fig. 3(c). LBCO95 in Fig. 3(b), (d) shows a similar diffuse scattering, but in addition two sharp superimposed CO-peaks.

\subsection{Normal State Magnetic Susceptibility}

The magnetic susceptibility $\chi$ of the normal state has proven a valuable tool for the analysis of the stripe phase in $\operatorname{LBCO}[11,16,17]$. This is particularly true for $x=1 / 8$ where bulk SC at $T_{c} \sim 3 \mathrm{~K}$ occurs much below the $\mathrm{CO}$ and SO transitions at $T_{\mathrm{CO}}=T_{\mathrm{LT}}=54 \mathrm{~K}$ and $T_{\mathrm{SO}}=42 \mathrm{~K}$, thus presenting a broad $T$-window for $\chi$-studies of the stripe phase above the bulk $T_{c}$. For LBCO95 the situation is less favorable, because at low fields $T_{c}=32 \mathrm{~K}$ is even slightly higher than $T_{\mathrm{CO}}=T_{\mathrm{LT}} \simeq 30 \mathrm{~K}$ [11]. However, $T_{c}$ can be pushed below $T_{\mathrm{CO}}$ and $T_{\mathrm{LT}}$ when a high magnetic field is applied. In addition, $T_{\mathrm{SO}}$ will shift to higher $T$ [16]. Corresponding results for LBCO95 and LBCZO95 for both $H \| c$ and $H \| a b$ are shown in Fig. 4. The 1 Tesla low-field data in Fig. 4(a) display an overview up to $375 \mathrm{~K}$. The 7 Tesla high-field data in Fig. 4(b) zoom in on the relevant low- $T$ region. There one can see that LBCO95 shows small anomalies at $\sim 33 \mathrm{~K}$ in both field directions. These anomalies are certainly connected to the stripe transition. But it remains unclear if they represent $T_{\mathrm{SO}}$ or $T_{\mathrm{CO}}$, or both, and if they have indeed shifted by $\sim 3 \mathrm{~K}$ in 7 Tesla, cf. Fig. 1 . In contrast, LBCZO95 lacks any anomaly that would indicate a sharp transition, and this is consistent with the broad LTO $\leftrightarrow$ LTLO transition in Fig. 2(a). At low $T$ LBCZO95 shows a Curie-type upturn caused by the effective paramagnetic moments induced by $\mathrm{Zn}[1,18]$.

\section{Discussion and Conclusions}

The key result that in LBCZO95 CO-peaks are not identifiable, while SO-peaks are enhanced, can either reflect an intrinsic effect of $\mathrm{Zn}$, or indicate sample specific properties such as a lower quality or a difference in the Ba content. With a mosaic of $0.01^{\circ}$ for LBCO95 and $0.018^{\circ}$ for LBCZO95, measured with XRD at the $(0,0,8)$ Bragg reflection at base temperature, both crystals demonstrate an excellent quality. Also the bulk SC transition of LBCZO95 is nearly as sharp as for LBCO95; see Fig. 1. To estimate the actual Ba content $x^{\prime}$ of LBCZO95, we apply the same strategy as in [11], which exploits the doping dependence of the HTT $\leftrightarrow$ LTO transition temperature $T_{\mathrm{HT}}$. As indicated in the inset of Fig. 4(a) the crystals $T_{\mathrm{HT}}$ differ by $\sim 18 \mathrm{~K}$. With $d T_{\mathrm{HT}} / d x \simeq-23 \mathrm{~K} / 0.01 \mathrm{Ba}$ and $d T_{\mathrm{HT}} / d z \simeq$ $8 \mathrm{~K} / 0.01 \mathrm{Zn}$, we calculate that LBCZO95 has a $\Delta x \simeq 0.004$ lower Ba content than LBCO95, consistent with the shift of 
Fig. 3 (Color online) Diffuse scattering around $(0,0,8)$ Bragg reflection and $( \pm 2 \delta, 0,8.5)$ $\mathrm{CO}$-peak for (c) $\mathrm{Zn}$-doped and (d) pure $\mathrm{La}_{1.905} \mathrm{Ba}_{0.095} \mathrm{CuO}_{4}$. (a) and (b) show $h$-scans along $(h, 0,8.5)$ as indicated by white arrows in (c) and (d)
Fig. 4 (Color online) Normal state $\chi(T)$ of pure and $\mathrm{Zn}$-doped $\mathrm{La}_{1.905} \mathrm{Ba}_{0.095} \mathrm{CuO}_{4}$ for (a) $H=1 \mathrm{~T}$ and (b) $7 \mathrm{~T}$ applied parallel $a b$-plane and $c$-axis. Inset: $d \chi / d T$ for $H \| a b$
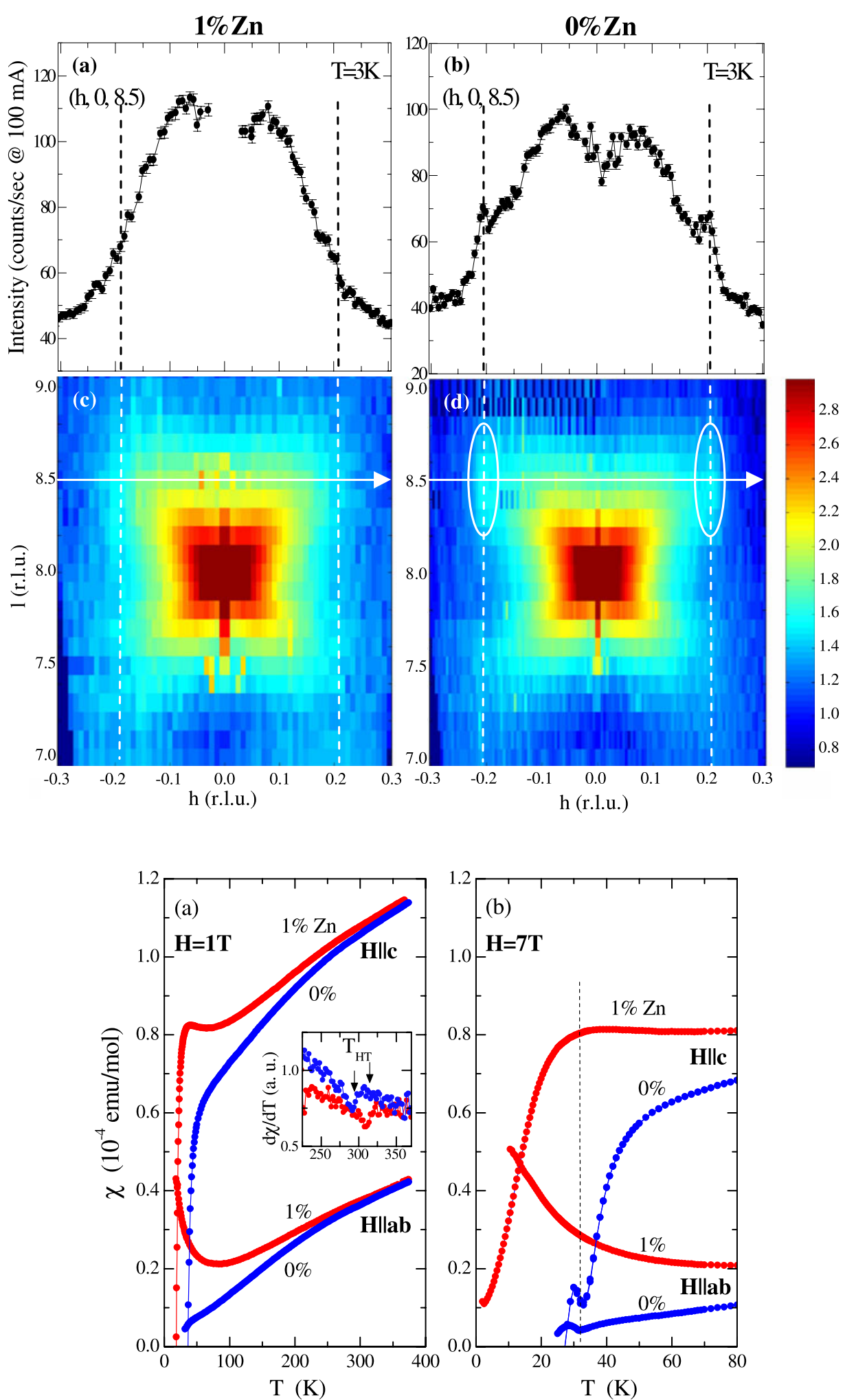

the SO-peak in Fig. 2(d). Absolute values were calculated to $x^{\prime} \simeq 0.099$ for LBCO95 and $x^{\prime} \simeq 0.095$ for LBCZO95 [11]. Does the absence of CO-peaks in LBCZO95 reflect that slightly lower $\mathrm{Ba}$ content, since $\mathrm{CO}$ rapidly decreases for $x<1 / 8$ [11]? Clearly not, since it is in disaccord with the observed increase of the SO. Hence, is it possible that CO is more sensitive to $\mathrm{Zn}$ impurities than $\mathrm{SO}$ ? Zn introduces spin $S=0$ vacancies to the $S=1 / 2$ spin lattice, but these do 
not cause strong perturbations of the AF correlations [18]. A sensible broadening of the SO-peaks only sets in for several percent $\mathrm{Zn}$ [19]. For charge carriers, $\mathrm{Zn}$ impurities act as localization centers [20], and because of their random distribution may cause significant disorder in the $\mathrm{CO}$ pattern. If the associated broadening of the CO-peaks is strong they may become undetectable, because at $x=0.095$, even without $\mathrm{Zn}, \mathrm{CO}$-peaks are much smaller than at $x=0.125$ [11]. According to the stripe model, $\mathrm{SO}$ and $\mathrm{CO}$ are intimately connected [7, 21]. Without charge stripes, which act as antiphase domain boundaries between spin stripes, the incommensurate SO would disintegrate, or become commensurate. Thus the question regarding LBCZO95 is: how disordered can the $\mathrm{CO}$ be until the SO collapses? Additional studies on LBCZO with $z<0.01$ and a more pronounced initial $(z=0) \mathrm{CO}$, such as at $x=1 / 8$, may tell.

\section{References}

1. Xiao, G., Cieplak, M.Z., Xiao, J.Q., Chien, C.L.: Phys. Rev. B 42, 8752 (1990)

2. Akoshima, M., Koike, Y., Watanabe, I., Nagamine, K.: Phys. Rev. B 62, 6761 (2000)

3. Watanabe, I., Akoshima, M., Koike, Y., Ohira, S., Nagamine, K.: Phys. Rev. B 62, 14524 (2000)

4. Adachi, T., Yairi, S., Koike, Y., Watanabe, I., Nagamine, K.: Phys. Rev. B 70, 060504R (2004)
5. Hirota, K., Yamada, K., Tanaka, I., Birgeneau, R.J.: Physica B 241, 817 (1998)

6. Kimura, H., Kofu, M., Matsumoto, Y., Hirota, K.: Phys. Rev. Lett. 91, 67002 (2003)

7. Tranquada, J.M., Sternlieb, B.J., Axe, J.D., Nakamura, Y., Uchida, S.: Nature 375, 561 (1995)

8. Fujita, M., Goka, H., Yamada, K., Tranquada, J.M., Regnault, L.P.: Phys. Rev. B 70, 104517 (2004)

9. Kivelson, S.A., Bindloss, I.P., Fradkin, E., Oganesyan, V., Tranquada, J.M., Kapitulnik, A., Howald, C.: Rev. Mod. Phys. 75, 1201 (2003)

10. Fujita, M., Enoki, M., Iikubo, S., Kudo, K., Kobayashi, N., Yamada, K.: arXiv:0903.5391 (2009)

11. Hücker, M., et al.: arXiv:1005.5191 (2010)

12. Hücker, M., v. Zimmermann, M., Debessai, M., Schilling, J.S., Tranquada, J.M., Gu, G.D.: Phys. Rev. Lett. 104, 057004 (2010)

13. Vajk, O., Greven, M., Mang, P., Lynn, J.: Sol. Solid State Commun. 126, 93 (2003)

14. Zhang, Y.Q., Qu, J.F., Wang, B.M., Xiang, X.Q., Li, X.G.: J. Appl. Phys. 102, 063910 (2007)

15. Wen, J.S., et al.: submitted

16. Hücker, M., Gu, G.D., Tranquada, J.M.: Phys. Rev. B 78, 214507 (2008)

17. Li, Q., Hücker, M., Gu, G.D., Tsvelik, A.M., Tranquada, J.M.: Phys. Rev. Lett. 99, 67001 (2007)

18. Hücker, M., Büchner, B.: Phys. Rev. B 65, 214408 (2002)

19. Kimura, H., et al.: Phys. Rev. B 59, 6517 (1999)

20. Hücker, M., Kataev, V., Pommer, J., Harraß, J., Hosni, A., Pflitsch, C., Büchner, B.: Phys. Rev. B 59, R725 (1999)

21. Kivelson, S.A., Fradkin, E., Emery, V.J.: Nature 393, 550 (1998) 